\title{
Erratum to: Using a discrete choice experiment to value the QLU- C10D: feasibility and sensitivity to presentation format
}

\author{
R. Norman ${ }^{1,2} \cdot$ R. Viney ${ }^{2}$ N. K. Aaronson ${ }^{3}$ J. E. Brazier ${ }^{4}$ D. Cella ${ }^{5}$ D. S. J. Costa ${ }^{6}$ \\ P. M. Fayers ${ }^{7,8}$ - G. Kemmler' S. Peacock ${ }^{10,11,12}$ • A. S. Pickard ${ }^{13}$ • D. Rowen ${ }^{4}$. \\ D. J. Street ${ }^{14}$ - G. Velikova ${ }^{15,16}$ • T. A. Young ${ }^{4}$ M. T. King ${ }^{6,17}$
}

Published online: 23 March 2017

(C) Springer International Publishing Switzerland 2017

\section{Erratum to: Qual Life Res (2016) 25:637-649 DOI 10.1007/s11136-015-1115-3}

In this article by R. Norman et al., the article by M. T. King et al. is cited as Reference 10, as 'Submitted' and 'Under Review'. However, the Reference 10 should appear with year, volume and page numbers as:

King et al., Quality of Life Research (2016); 25(3):625-636.
Also an error was found in Table 1 in the reported wording of the Physical Functioning item. The error and correction are described below. The error was limited to Table 1. The survey described in the paper used the correct labelling, and the validity of the analysis is therefore unaffected by the error.

The online version of the original article can be found under doi:10.1007/s11136-015-1115-3.

\section{R. Norman}

Richard.norman@curtin.edu.au

1 School of Public Health, Curtin University, Perth, Australia

2 Centre for Health Economics Research and Evaluation (CHERE), University of Technology Sydney (UTS), Sydney, Australia

3 The Netherlands Cancer Institute, Amsterdam, The Netherlands

4 School of Health and Related Research, University of Sheffield, Sheffield, South Yorkshire, UK

5 Department of Medical Social Sciences, Feinberg School of Medicine, Northwestern University, Chicago, IL, USA

6 Psycho-Oncology Cooperative Research Group (PoCoG), University of Sydney, Sydney, Australia

7 Institute of Applied Health Sciences, University of Aberdeen, Aberdeen, UK

8 Department of Cancer Research and Molecular Medicine, Norwegian University of Science and Technology (NTNU), Trondheim, Norway
Innsbruck Medical University, Innsbruck, Austria

10 Faculty of Health Sciences, Simon Fraser University, Vancouver, Canada

11 Canadian Centre for Applied Research in Cancer Control (ARCC), Vancouver, Canada

12 British Columbia Cancer Agency, Vancouver, Canada

13 Department of Pharmacy Systems, Outcomes and Policy, College of Pharmacy, University of Illinois at Chicago, Chicago, IL, USA

14 School of Mathematical and Physical Sciences, University of Technology Sydney, Sydney, Australia

15 Leeds Institute of Cancer and Pathology, University of Leeds, Leeds, UK

16 St James's Hospital, Leeds, UK

17 Central Clinical School, Sydney Medical School, University of Sydney, Sydney, Australia 
Correction to Table 1: Level labels for the Physical Functioning dimension

Incorrect label origi- Correct label nally reported

Level 1

No trouble taking a short walk outside

No trouble taking a of the house

Level 2

At least a little trouble taking a long walk short walk outside of the house, and no trouble taking a

At least a little trouble taking a long walk but no trouble taking a short walk outside long walk

Level 3

At least a little trouble taking a short walk outside of the house, and at least a little trouble taking a long walk

Level 4 Quite a bit or very much trouble taking a short walk outside the house the house

A little trouble taking a short walk outside the house

Quite a bit or very much trouble taking a short walk outside the house 